\title{
Antidiabetes Agents against Sars-Cov-2 Infection
}

\author{
Antonio Vitiello $^{1} \cdot$ Francesco Ferrara $^{1}$ \\ Accepted: 20 October 2020 / Published online: 24 October 2020 \\ (C) Springer Nature Switzerland AG 2020
}

\begin{abstract}
People with chronic diseases represent a population at major risk of infection and complications from Sars-Cov-2 (COVID-19). Diabetes represents one of the most important comorbidities related to the severity of viral infection caused by the new Sars-Cov2. Diabetes patients have a higher risk of serious complications caused by Sars-Cov-2 infection, such as severe acute respiratory syndrome, a hyperinflammatory state associated with multi-organ dysfunction. Given the importance of the link between COVID-19 and diabetes, it is essential to better manage glycemic normalization and infection in this category of patients to avoid serious complications. However, for some antidiabetes agents, there is evidence of efficacy against Sars-Cov-2 extra glycemic normalization. The objective of this article is to provide an overview of the potential therapeutic benefits to fight Sars-Cov-2 infection with antidiabetic agents.
\end{abstract}

Keywords Diabetes $\cdot$ COVID-19 $\cdot$ SARS-CoV-2 $\cdot$ Infection $\cdot$ Antidiabetes agents

\section{Introduction}

\section{COVID-19 Infection}

The global pandemic caused by the new Sars-Cov-2 (COVID19), recorded its first cases in China in November 2019, and within a few months, became a worldwide problem, representing a health emergency with few precedents in human history. As of now, 5.8 million positive cases and 360,000 deaths were recorded with over 250 countries affected [1]. COVID-19 infection is divided into three phases, the first slightly symptomatic or asymptomatic, the second and third more severe, with increased inflammatory status and cytokinic cascade causing multi-organ dysfunction resulting in fatal lung injury. Studies have shown that people with pre-existing chronic diseases such as diabetes are at increased risk of infection and serious complications. To date, there are no direct antivirals and effective vaccines against Sars-Cov-2 [2, 3].

This article is part of the Topical Collection on Covid-19

Francesco Ferrara

francesco.ferrara@uslumbria1.it

Antonio Vitiello

antonio.vitiello2@uslumbria1.it

1 Pharmaceutical Department, Usl Umbria 1, A.Migliorati street, 06132 Perugia, Italy

\section{COVID-19 and Diabetes Mellitus}

Diabetes is an important risk factor for viral, bacterial, and fungal infections. During the current global COVID-19 pandemic, diabetes has been identified as a primary risk factor for the development of severe Sars-Cov-2 viral infection pneumonia. Epidemiological data show that the risk of a fatal outcome of COVID-19 is up to 50\% higher in patients with diabetes than in those without diabetes. There are many factors that may increase the risk for diabetes patients with COVID-19; among them, the presence of a deficient immune system, a dysfunctional coagulation cascade, a hyperactive inflammatory state, in the elderly patient with diabetes, there is also almost always a cardiovascular disease that could explain the more severe outcome of COVID-19 infection. In addition, the Sars-Cov-2 virus uses the angiotensin-converting enzyme 2 (ACE2) protein to enter the lung epithelial cells [4-6]. ACE2 has protective effects especially with regard to inflammation of the respiratory tract. COVID-19 infection in the most severe phases (two and three) reduces the expression of ACE2; this is one of the causes of hyperinflammation and respiratory failure. In the diabetic patient, ACE2 expression is altered, and this may cause an even more complicated clinical situation in the COVID-19 patient; moreover, the viral infection itself may cause a worsening of the diabetic disease with a glycemia that is difficult to control. [7-9] Another fundamental aspect to consider is that the DPP-4 protein (therapeutic target of gliptins) has been identified as a functional 
receptor for the virus responsible for MERS [10], and to date, it is not certain whether it is also a functional receptor for SarsCov-2. In patients with diabetes, the expression of DPP-4 is altered and is responsible for overactive inflammation, which could further complicate the clinical situation [11]. Based on the considerations expressed, it emerges that the management of the patient with diabetes and COVID-19 positive is extremely delicate. The recommendations indicate that drug treatment for diabetes should not be discontinued during viral infection, if there are no particular contraindications; however, for some of them, there is evidence in the literature that shows their potential additional therapeutic role against COVID-19 infection.

\section{Antidiabetes Agent and Potential Therapeutic Role against COVID-19}

Some antidiabetes drugs have shown pleiotropic activity added to the glycemic normalization (Table 1); these pieces of evidence of efficacy have been confirmed by in vitro studies and on epidemics similar to COVID-19, such as SARS and MERS; if these pieces of evidence will be confirmed by clinical and epidemiological data for the current pandemic, they may represent additional weapons to fight COVID-19 infection in the diabetic patient [12].

\section{Metformin}

It is now known that in the most severe stages of COVID-19 infection, an overactive and uncontrolled inflammatory system triggered by an uncontrolled cytokinic cascade is responsible for multi-organ dysfunction and fatal lung injury. The use of metformin has shown a decrease in inflammatory markers through different modes of action. Evidence has shown that metformin causes a decrease in the production of reactive oxygen species (ROS) by inhibition of nicotinamide adenine dinucleotide phosphate $\mathrm{NAD}(\mathrm{P}) \mathrm{H})$ oxidase and the respiratory mitochondrial chain; other studies have suggested

Table 1 Agents antidiabetes and pleiotropic activity added to the glycemic normalization

Antidiabetes agents and extra "glycemic regulation" effects against COVID-19

\begin{tabular}{ll}
\hline Metformin & Anti-inflammatory \\
Gliptins & Anti-inflammatory \\
GLP-1RA & Anti-inflammatory \\
Pioglitazone & Reduce pro-inflammatory cytokines \\
SGLT2 inhibitors & Reducing lactate levels \\
Sulfonylureas & Improvement in platelet function \\
\hline
\end{tabular}

that metformin suppresses inflammatory response by inhibition of nuclear factor $\mathrm{KB}(\mathrm{NF} \kappa \mathrm{B})$ through pathways dependent on AMP-activated protein kinase (AMPK) and through the blockade of 3-kinase phosphoinosis (PI3K)-Akt [13]. Other studies have shown that metformin reduces the production of NO, prostaglandin E2 (PGE2), and pro-inflammatory cytokines (interleukin (IL)- $1 \beta$, IL-6, and tumor necrosis factor (TNF)- $\alpha$ ) by inhibition of NfkB activation in macrophages. Finally, there is evidence that metformin also suppresses inflammatory mediators specifically in lung tissue [14] damaged by viral infections such as SARS and MERS; however, its use in severe patients with COVID-19 should be carefully assessed in light of the risks of lactic acidosis in patients with renal dysfunction, which may be present in more severe COVID-19 cases [15]. Metformin use has shown a decrease in inflammatory markers in patients with SARS and MERS, but seems to have an additional advantage on viral infections, particularly on hepatitis $\mathrm{C}$ virus (HCV) $[16,17]$ and RNA viruses such as Sars-Cov-2. In addition, according to studies, metformin therapy reduces liver fibrosis in $\mathrm{HCV}$ patients [18]; therefore, a protective role on the liver could be hypothesized even in the most severe stages caused by Sars-Cov 2 infection.

\section{Gliptins}

The DPP-4 protein has several actions; in particular, it plays an important role in immune regulation by activating $\mathrm{T}$ cells and upregulating CD86 expression and NF-jB pathway, and it is responsible for increasing inflammation in patients with diabetes. It should also be emphasized that the enzymatic activity of DPP4 causes cleavage and may affect the function of several cytokines, chemokines, and growth factors. Some studies have suggested that a higher mortality rate and complications in people with diabetes and infected with MERS-CoV may be associated with a dysregulated DPP4-mediated immune response.

DPP4 inhibitors (sitagliptin, vildagliptin, saxagliptin, etc.) are drugs of undoubted therapeutic efficacy in the treatment of diabetes. Recently, this class of drugs has been associated with pleiotropic therapeutic benefits that go beyond their lowering of glucose. However, the effects of DPP4 inhibition on the immune system and inflammation are controversial and not fully understood.

However, a recent meta-analysis has shown that upper respiratory tract infections do not significantly increase with DPP-4 inhibitory treatment. Another aspect to consider is that the DPP4 protein is expressed in many cells including alveolar epithelium and inflammatory cells. MERS-CoV uses DPP IV to enter host cells [19], but it is not known whether Sars-Cov-2 also uses the same protein to enter the cell, in addition to ACE2. If this is demonstrated, the use of gliptins could decrease the risk of Sars-Cov-2 infection but this has not been demonstrated so far. The potential benefit in the treatment of Sars-Cov-2 infection with DPP IV inhibitors remains to be further 
investigated. To date, it is not totally clear whether DPP IV inhibition may play an important role in controlling the inflammatory status in patients with diabetes and COVID-19 infection; however, it may represent a potential target to prevent and reduce the risk and progression of acute respiratory tract complications that the patient with diabetes may add to COVID-19 infection [20].

\section{GLP-1RA}

Similar to metformin, the effects of reducing inflammatory markers are also known for GLP-1RA; these well-known antidiabetes drugs have also shown potential therapeutic benefit in acute lung injury [21]. Recent studies have also shown that GLP-1 agonists possess anti-inflammatory and antiproliferative functions. In vitro studies have also shown that an agent belonging to the GLP-1RA class such as exenatide significantly increased the level of IL-10 and decreased both TNF- $\alpha$ and IL-1 $\beta$ in monocytes/macrophages [22]. In addition, the exenatide increased and reduced the LPS-induced expression of iNOS. However, the available data are limited to experimental models and their benefit is still to be studied.

\section{Pioglitazone}

Pioglitazone belongs to thiazolidinediones (TZD) drugs, used for many years for the treatment of diabetes. Evidence has shown that pioglitazone can have an anti-inflammatory effect as it has been tested through the high-sensitivity reactive $\mathrm{C}$ protein within a short time after the start of therapy. In particular, it has been shown that pioglitazone $(30-45 \mathrm{mg}$ /day for 3 months) can reduce IL- 6 and TNF, and after 4 months of therapy ( $45 \mathrm{mg} /$ day), treatment with pioglitazone reduced the monocyte gene and protein expression of IL- $1 \mathrm{~b}, \mathrm{IL}-6$, and IL-8 and lymphocyte IL-2, IL-6, and IL-8. It has also been reported that pioglitazone inhibits the secretion of pro-inflammatory cytokines (e.g., IL-1b, IL-6, and IL-8) and may increase the antiinflammatory ones (e.g., IL-4 and IL-10) in astrocytes [23]. Finally, pioglitazone has been reported to have a direct effect in reducing inflammation and pulmonary fibrosis [24].

\section{SGLT2 Inhibitors}

Sodium-glucose inhibitor drugs cotransporter-2 (SGLT2) are very effective in the treatment of diabetes and heart-related problems. SGLT2 inhibitors have been reported to prevent the release of pro-inflammatory cytokines such as interleukin 6 . In addition, during Sars-Cov-2 infection, the level of serum lactate dehydrogenase (LDH) increases; in fact, COVID-19 infection can increase LDH in the bloodstream by disrupting organs and cells, causing metabolic acidosis. In addition, elevated lactate levels increase the release of pro-inflammatory cytokines and cellular oxidative stress. Dapagliflozin is an SGLT2 inhibitor and has been shown to be effective in reducing lactate levels [25]. Dapagliflozin can also prevent cell damage and death by both increasing tissue oxygenation and decreasing lactate levels. Dapagliflozin has been shown to have a cytoprotective effect. In addition, SGLT2 inhibitors increase the level of ACE2 which is known to play a protective role especially in the more severe stages of COVID-19 infection [26].

\section{Sulfonylureas}

Sulfonylureas have also been shown to have antiinflammatory effects. In the most severe stages of COVID19 infection, and especially in patients with diabetes, there is a dysfunction of coagulation and platelet aggregation, an improvement in platelet function, and reduced thromboxane activation in the metabolic pathway have been reported for this class of drugs [27].

\section{Conclusions}

The COVID-19 global pandemic represents one of the greatest health challenges in human history. Patients with comorbidities such as diabetes may be at greater risk of complications if infected with COVID-19. The treatment of diabetes in a COVID-19 patient must be carefully managed to avoid serious adverse reactions, but for some drugs, there is evidence of extrapancreatic pleiotropic effects and extra glycemic normalization that can be an added value in the fight against COVID-19 infection.

Authors Contribution Antonio Vitiello: Conceptualization, writingoriginal draft, methodology, writing - original draft. Francesco Ferrara: Writing - review and editing, supervision, validation.

\section{Compliance with Ethical Standards}

Competing Interest The authors declare that they have no conflict of interest.

Ethics Approval Not applicable.

Consent to Participate Not applicable.

Consent for Publication Not applicable.

\section{References}

1. World Health Organization HO. Coronavirus disease 2019 (COVID-19) situation report. 2020. Available from https://www. who.int/emergencies/diseases/novel-coronavirus-2019/situationreports.

2. Lin L, Lu L, Cao W, Li T. Hypothesis for potential pathogenesis of SARS-CoV-2 infection-a review of immune changes in patients 
with viral pneumonia. Emerg Microbes Infect. 2020;9(1):727-32. https://doi.org/10.1080/22221751.2020.

3. Vitiello A, Ferrara F, Pelliccia C, Granata G, La Porta R. Cytokine storm and colchicine potential role in fighting SARS-CoV-2 pneumonia. Ital J Med. 2020;14(2):88-94. https://doi.org/10.4081/itjm. 2020.1284 .

4. Vitiello A, Ferrara F. Pharmacological agents to therapeutic treatment of cardiac injury caused by Covid-19 [published online ahead of print, 2020 Sep 28]. Life Sci. 2020;262:118510. https://doi.org/ 10.1016/j.lfs.2020.118510.

5. Ferrara F. Antirheumatic in SARS-CoV-2: benefit or risk? Ital J Med. 2020;14(2):114-5. https://doi.org/10.4081/itjm.2020.1290.

6. Vitiello A, La Porta R, Ferrara F. Sacubitril, valsartan and SARSCoV-2. BMJ Evid Based Med. 2020. https://doi.org/10.1136/ bmjebm-2020-111497.

7. Vitiello A, Ferrara F. Therapeutic strategies for SARS-CoV-2 acting on ACE-2 [published online ahead of print, 2020 Sep 30]. Eur J Pharm Sci. 2020;156:105579. https://doi.org/10.1016/j.ejps.2020. 105579.

8. Ferrara F, Granata G, Pelliccia C, La Porta R, Vitiello A. The added value of pirfenidone to fight inflammation and fibrotic state induced by SARS-CoV-2 : anti-inflammatory and anti-fibrotic therapy could solve the lung complications of the infection? Eur J Clin Pharmacol. 2020;76(11):1615-8. https://doi.org/10.1007/s00228020-02947-4.

9. Yang JK, Lin SS, Ji XJ, Guo LM. Binding of SARS coronavirus to its receptor damages islets and causes acute diabetes. Acta Diabetol. 2010;47(3):193-9. https://doi.org/10.1007/s00592-009-0109-4.

10. Raj VS, Mou H, Smits SL, Dekkers DH, Müller MA, Dijkman R, et al. Dipeptidyl peptidase 4 is a functional receptor for the emerging human coronavirus-EMC. Nature. 2013;495(7440):251-4. https://doi.org/10.1038/nature12005.

11. Roy S, Mazumder T, Banik S. The association of cardiovascular diseases and diabetes mellitus with COVID-19 (SARS-CoV-2) and their possible mechanisms. SN Compr Clin Med. 2020;2:1077-82. https://doi.org/10.1007/s42399-020-00376-Z.

12. Vitiello A, Pelliccia C, Ferrara F. COVID-19 patients with pulmonary fibrotic tissue: clinical pharmacological rational of antifibrotic therapy [published online ahead of print, 2020 Aug 27]. SN Compr Clin Med. 2020:1-4. https://doi.org/10.1007/s42399-020-00487-7.

13. Li SN, Wang X, Zeng QT, Feng YB, Cheng X, Mao XB, et al. Metformin inhibits nuclear factor kappaB activation and decreases serum high-sensitivity C-reactive protein level in experimental atherogenesis of rabbits. Heart Vessel. 2009;24(6):446-53. https://doi. org/10.1007/s00380-008-1137-7.

14. Penlioglou T, Papachristou S, Papanas N. COVID-19 and diabetes mellitus: may old anti-diabetic agents become the new philosopher's stone? Diabetes Ther. 2020;11:1195-7. https://doi.org/10. 1007/s13300-020-00830-0.

15. Tsai WL, Chang TH, Sun WC, et al. Metformin activates type I interferon signaling against $\mathrm{HCV}$ via activation of adenosine monophosphate-activated protein kinase. Oncotarget. 2017;8(54): 91928-37. https://doi.org/10.18632/oncotarget.20248.

16. Iranshahy M, Rezaee R, Karimi G. Hepatoprotective activity of metformin: a new mission for an old drug? Eur J Pharmacol. 2019;850:1-7. https://doi.org/10.1016/j.ejphar.2019.02.004.
17. Iacobellis G. COVID-19 and diabetes: can DPP4 inhibition play a role? Diabetes Res Clin Pract. 2020;162:108125. https://doi.org/10. 1016/j.diabres.2020.108125.

18. Feng Y, Wang L, Ma X, Yang X, Don O, Chen X, et al. Effect of hCMSCs and liraglutide combination in ALI through cAMP/PKAc/ $\beta$-catenin signaling pathway. Stem Cell Res Ther. 2020;11:2. https://doi.org/10.1186/s13287-019-1492-6.

19. Kawanami D, Matoba K, Takeda Y, Nagai Y, Akamine T, Yokota $\mathrm{T}$, et al. SGLT2 inhibitors as a therapeutic option for diabetic nephropathy. Int J Mol Sci. 2017;18(5):1083. https://doi.org/10.3390/ ijms 18051083.

20. Violi F, De Mattia GC, Alessandri C, Perrone A, Vezza E. The effects of gliclazide on platelet function in patients with diabetes mellitus. Curr Med Res Opin. 1982;8(3):200-3. https://doi.org/10. 1185/03007998209112384.

21. Park CS, Bang BR, Kwon HS, Moon KA, Kim TB, Lee KY, et al. Metformin reduces airway inflammation and remodeling via activation of AMP-activated protein kinase. Biochem Pharmacol. 2012;84(12):1660-70. https://doi.org/10.1016/j.bcp.2012.09.025.

22. DeFronzo R, Fleming GA, Chen K, Bicsak TA. Metforminassociated lactic acidosis: current perspectives on causes and risk. Metabolism. 2016;65(2):20-9. https://doi.org/10.1016/j.metabol. 2015.10.014.

23. Wang C, Chen X, Ding X, He Y, Gu C, Zhou L. Exendin-4 promotes beta cell proliferation via PI3k/Akt signalling pathway. Cell Physiol Biochem. 2015;35:2223-32. https://doi.org/10.1159/ 000374027.

24. Bułdak $Ł$, Machnik G, Jakub Buldak R, et al. Erratum to "Exenatide (a GLP-1 agonist) expresses anti-inflammatory properties in cultured human monocytes/macrophages in a protein kinase a and B/Akt manner". Pharmacol Rep. 2019;71:985-6. https://doi. org/10.1016/j.pharep.2019.05.019.

25. Qiu D, Li XN. Pioglitazone inhibits the secretion of proinflammatory cytokines and chemokines in astrocytes stimulated with lipopolysaccharide. Int J Clin Pharmacol Ther. 2015;53(9):746-52. https://doi.org/10.5414/CP202339.

26. Aoki Y, Maeno T, Aoyagi K, Ueno M, Aoki F, Aoki N, et al. Pioglitazone, a peroxisome proliferator-activated receptor gamma ligand, suppresses bleomycin-induced acute lung injury and fibrosis. Respiration. 2009;77(3):311-9. https://doi.org/10.1159/ 000168676

27. Couselo-Seijas M, Agra-Bermejo RM, Fernández AL, MartínezCereijo JM, Sierra J, Soto-Pérez M, et al. High released lactate by epicardial fat from coronary artery disease patients is reduced by dapagliflozin treatment. Atherosclerosis. 2020;292:60-9. https:// doi.org/10.1016/j.atherosclerosis.2019.11.016.

Publisher's Note Springer Nature remains neutral with regard to jurisdictional claims in published maps and institutional affiliations.

The authors certify that the manuscript is original, never submitted to other journals for publication before. All authors contributed equally to the manuscript and had the opportunity to revise and approve the final text. 